Mens

Revue d'histoire intellectuelle et culturelle

mens

\title{
Antoine Gérin-Lajoie : une jeunesse romantique et un idéal politique au cours des difficiles années de l'Union
}

\section{Gilles Gallichan}

Volume 19, numéro 1-2, automne 2018, printemps 2019

Les années 1840 : rupture ou réarticulation des possibles ?

URI : https://id.erudit.org/iderudit/1070071ar

DOI : https://doi.org/10.7202/1070071ar

Aller au sommaire du numéro

Éditeur(s)

Centre de recherche en civilisation canadienne-française

ISSN

1492-8647 (imprimé)

1927-9299 (numérique)

Découvrir la revue

Citer cet article

Gallichan, G. (2018). Antoine Gérin-Lajoie : une jeunesse romantique et un idéal politique au cours des difficiles années de l'Union. Mens, 19(1-2), 117-137. https://doi.org/10.7202/1070071ar

\section{Résumé de l'article}

Cet article met en relief les premières années de la carrière d'Antoine Gérin-Lajoie et les premiers pas de son engagement social et national. Né en 1824, il fut témoin, sans être acteur, des grandes années patriotes et des insurrections de 1837-1838. Sa formation scolaire s'accompagne d'éveils culturels et d'élans littéraires qui le placent parmi les auteurs du courant romantique québécois. L’itinéraire du jeune Gérin-Lajoie, dans la décennie 1840, illustre les espoirs et les difficultés de sa génération. Il cherche brièvement fortune aux États-Unis, puis il revient au pays, préférant servir ses concitoyens par le journalisme et l'écriture. Son idéal politique est déchiré par la rupture entre L.-H. LaFontaine, qui tente de composer avec les contraintes et les injustices de l'Union, et L.-J. Papineau, revenu d'exil, qui condamne le régime colonial et se tourne vers l'annexionnisme. Gérin-Lajoie trouve dans la fonction publique, plus que dans le journalisme, un moyen de gagner sa vie et de fonder une famille. Son oeuvre littéraire, tant du côté de la poésie et du roman que de l'histoire, lui permet cependant de travailler au relèvement national. Homme de lettres et de réflexion, il figure, avec d'autres Canadiens de son époque (Étienne Parent, F.-X. Garneau, P.-J-O. Chauveau, etc.), parmi ceux qui, malgré le long hiver colonial de l’Union, ont travaillé à maintenir l'existence du Canada français et qui ont souhaité son épanouissement.
Ce document est protégé par la loi sur le droit d'auteur. L’utilisation des services d’Érudit (y compris la reproduction) est assujettie à sa politique d'utilisation que vous pouvez consulter en ligne.

https://apropos.erudit.org/fr/usagers/politique-dutilisation/ 


\section{Antoine Gérin-Lajoie: une jeunesse romantique et un idéal politique au cours des difficiles années de l'Union}

Gilles Gallichan

Historien et bibliothécaire retraité

\section{Résumé}

Cet article met en relief les premières années de la carrière d'Antoine Gérin-Lajoie et les premiers pas de son engagement social et national. Né en 1824, il fut témoin, sans être acteur, des grandes années patriotes et des insurrections de 1837-1838. Sa formation scolaire s'accompagne d'éveils culturels et d'élans littéraires qui le placent parmi les auteurs du courant romantique québécois. L'itinéraire du jeune Gérin-Lajoie, dans la décennie 1840, illustre les espoirs et les difficultés de sa génération. Il cherche brièvement fortune aux États-Unis, puis il revient au pays, préférant servir ses concitoyens par le journalisme et l'écriture. Son idéal politique est déchiré par la rupture entre L.-H. LaFontaine, qui tente de composer avec les contraintes et les injustices de l'Union, et L.-J. Papineau, revenu d'exil, qui condamne le régime colonial et se tourne vers l'annexionnisme. Gérin-Lajoie trouve dans la fonction publique, plus que dans le journalisme, un moyen de gagner sa vie et de fonder une famille. Son œuvre littéraire, tant du côté de la poésie et du roman que de l'histoire, lui permet cependant de travailler au relèvement national. Homme de lettres et de réflexion, il figure, avec d'autres Canadiens de son époque (Étienne Parent, F.-X. Garneau, P.-J.-O. Chauveau, etc.), parmi ceux qui, malgré le long hiver colonial de l'Union, ont travaillé à maintenir l'existence du Canada français et qui ont souhaité son épanouissement. 


\section{Abstract}

This article highlights the first years of Antoine Gerrin-Lajoie's career. His itinerary in the 1840s illustrates the hopes and difficulties of his generation. His political ideal was torn apart by the break between L.-H. LaFontaine and L.-J. Papineau. As a man of letters, he figures, with other Canadians of his time (Etienne Parent, F-X. Garneau, P.-J.-O. Chauveau, etc.), among those who, despite the Union, worked to maintain and enrich the existence of French Canada.

Antoine Gérin-Lajoie (1824-1882) fut un brillant journaliste, écrivain et fonctionnaire, apprécié par ses contemporains et qui demeure un auteur important du XIX ${ }^{\mathrm{e}}$ siècle québécois ${ }^{1}$. Il appartient à la génération qui a atteint l'âge adulte pendant la décennie 1840 . Les événements de ces années cruciales dans l'histoire du Québec ont marqué son itinéraire professionnel, ses choix politiques, ses sentiments nationaux et sa vision du pays. On les retrouve, sous plusieurs aspects, intégrés à son œuvre. Il a d'ailleurs consacré à cette décennie un essai historique, qu'il qualifiait de «mémoires» et qu’il considérait comme son témoignage personnel, quoiqu’impartial, de cette époque ${ }^{2}$. Pendant ces années, sa formation et sa carrière ont

1 Sur Antoine Gérin-Lajoie et son époque, nous devons beaucoup aux recherches de René Dionne, qui a publié, en 1978, la biographie la plus complète du personnage et qui demeure, à ce jour, un point de départ important pour toute étude sur l'homme et son temps (René Dionne, Antoine Gérin-Lajoie, homme de lettres, Sherbrooke, Éditions Naaman, 1978). La bibliographie de cet ouvrage constitue une référence générale de base sur le personnage. On consultera aussi Henri-Raymond Casgrain, A. Gérin-Lajoie d'après ses mémoires, Montréal, Éditions Beauchemin, 1912 et le tome II de ses Euvres complètes, Montréal, Beauchemin \& Valois, 1885, p. 431-542. On peut aussi consulter Jean-Charles Falardeau, "Gérin-Lajoie, Antoine», dans Dictionnaire biographique du Canada, vol. 11, Québec, Université Laval; Toronto, University of Toronto, 2003, [En ligne], [http://www.biographi.ca/fr/bio/gerin_lajoie_antoine_11F.html] (15 janvier 2018). Selon les spécialistes, cette dernière biographie n’apporte pas de nouveaux éléments aux connaissances sur Gérin-Lajoie.

2 Antoine Gérin-Lajoie, Dix ans au Canada de 1840 à 1850: histoire de l'établissement du gouvernement responsable, Québec, L.-J. Demers, 1888-1891, p. 9-10. 
été soumises à des contraintes partagées par les jeunes Canadiens qui ont succédé à la cohorte des insurgés de 1837 et qui ont subi les conséquences politiques, culturelles et sociales de l'Union de $1840^{3}$. Un regard sur son parcours d'enfance et de jeunesse nous révèle une part de sa personnalité et de ses aspirations. Mais, c'est le contexte politique de l'époque qui l'a façonné en freinant ses ambitions, tout en forgeant ses idéaux et en l'orientant vers des chemins plus réalistes.

Antoine Gérin-Lajoie est né en 1824, à Yamachiche. Il a donc été contemporain des événements de 1837-1838, tout en étant trop jeune pour y participer. Fils de cultivateur, élève brillant, rêveur et ambitieux, il fait partie des privilégiés qui, à cette époque, ont eu la chance de fréquenter un collège et d'améliorer leur statut social et économique. Or le contexte politique de l'Union, le difficile « hiver colonial» des années 1840, selon le mot de l'historien Louis-Georges Harvey, a constitué une entrave à l'élan de ceux qui débutaient dans la vie à ce moment charnière de l'histoire. En même temps, cette décennie a aussi représenté un laboratoire de "possibles " pour l'émergence des lettres, de la presse, du monde associatif, un changement dans le discours politique et l'amorce de certaines réformes structurelles devenues urgentes. Antoine Gérin-Lajoie est l'exemple $\mathrm{du}$ jeune homme intelligent ayant eu la chance de recevoir une bonne éducation et capable de faire fructifier le bagage de culture que l'on avait mis à sa portée. À travers l'itinéraire de sa jeunesse et le début de sa carrière, on peut comprendre le contexte de son époque et voir en lui un témoin de son temps.

3 Sur les conséquences de l'Union au Canada français, le chercheur trouvera une très abondante bibliographie depuis les ouvrages pionniers de Louis-Philippe Turcotte, Le Canada sous l'Union, 1841-1867, t. I et II, Québec, Imprimerie du Canadien, 1871-1872, ou Laurent-Olivier David, L'Union des deux Canadas, 1841-1867, Montréal, É. Sénécal, 1898, jusqu'aux travaux plus récents d’Yvan Lamonde, Histoire sociale des idées, 1760-1896, Montréal, Éditions Fides, 2000, p. 283-358 et d'Éric Bédard, Survivance : histoire et mémoire du XIX siècle canadienfrançais, Montréal, Éditions du Boréal, 2017, en particulier les chapitres 1 et 2, p. 9-46. 


\section{L'enfance et la formation d'un futur écrivain}

Antoine Gérin-Lajoie est l'arrière-petit-fils d'un sergent de marine, Jean Jarrin dit Lajoie, venu en Nouvelle-France vers 1750 pour défendre la colonie contre l'invasion britannique et qui s'est établi dans la vallée du lac Saint-Pierre pour y prendre racine après la Conquête. Cet ancêtre eut trois fils, dont l'un, André, a repris la terre familiale. Il fut le père d'Antoine Gérin, qui épousa Marie-Amable Gélinas. Le couple s'est établi à Yamachiche, sur un nouveau lot du village. Par la mort prématurée d'un premier enfant, leur fils Antoine Gérin-Lajoie est devenu l'aîné d'une famille de 16 enfants. Le jeune Antoine a grandi dans un milieu stable et stimulant. Ses parents lui ont transmis des valeurs fondamentales, dont celles de l'amour de la terre et de la patrie ${ }^{4}$.

Antoine est perçu comme un enfant tranquille, de caractère facile et hypersensible ${ }^{5}$. Comme une partie des jeunes enfants canadiens de son âge, Antoine Gérin-Lajoie profite, dans les années 1820, d'un réveil collectif et d'un premier essor, encore timide, de l'instruction publique. Lannée de sa naissance, en 1824, un comité spécial de la Chambre d'assemblée du Bas-Canada recommande l'adoption d'une loi scolaire ${ }^{6}$. Cette réforme favorise la création d'écoles dans les paroisses et villages de la province. Quelques années plus tard, celles-ci deviennent des écoles de syndics avec l'adoption de la loi de $1829^{7}$. Ces

4 Falardeau, "Gérin-Lajoie, Antoine»; Louvigny de Montigny, Antoine GérinLajoie, Toronto, Ryerson Press, 1925, p. 2-3. La grande émotivité qui caractérisait Gérin-Lajoie lui viendrait de sa mère selon son biographe (Dionne, Antoine Gérin-Lajoie, homme de lettres, p. 30-31).

5 [Léon Gérin], Antoine Gérin-Lajoie: la résurrection d'un patriote canadien, Montréal, Éditions du Devoir, 1925, p. 10.

6 Rapport du comité spécial de la Chambre d'assemblée du Bas-Canada nommé pour s'enquérir de l'état actuel de l'éducation dans la province du Bas-Canada. Ordonné le $2^{e}$ février 1824, par la Chambre d'assemblée du Bas-Canada qu'il soit imprimé, Québec, T. Cary, 1824, [En ligne], [http://eco.canadiana.ca/view/ oocihm.21171/3?r=0\&s=1] (15 janvier 2018).

7 Jean-Pierre Proulx, La genèse de l'école publique et la démocratie scolaire au Québec: les écoles de syndics 1814-1838, Québec, Presses de l'Université Laval, 2014. Le 
lois ont connu des difficultés d'application, des limites et des lacunes, mais sans cette volonté politique de mise en valeur de l'éducation au cours des années 1820 et 1830 , bien des petites écoles rurales n'auraient pas existé. Le jeune Gérin-Lajoie fait donc ses premières classes à l'école de son village et il reçoit quelques éléments de latin auprès d'un maître à l'école, dite supérieure, de Yamachiche. Il apprend vite et bien et se fait la réputation d'un enfant surdoué 8 .

Celui qui, le premier, aura le mérite de «découvrir» son talent est Sévère-Nicolas Dumoulin, le curé de son village depuis 1825. Le curé Dumoulin est un ancien missionnaire à la Rivière-Rouge ${ }^{9}$ et un pasteur actif. Il profite de toutes les occasions pour organiser, dans sa paroisse, des manifestations religieuses ou patriotiques qui ont pu marquer l'imagination du jeune Antoine. L'abbé Dumoulin, qui a questionné l'enfant et évalué ses aptitudes, convainc les parents d'Antoine de l'envoyer faire son cours classique à Nicolet; il paiera de ses deniers un trimestre de pension par année au Séminaire.

À l'été 1837, Antoine a 13 ans au moment où le Bas-Canada entre en insurrection contre le gouvernement colonial anglais. Il est possible qu'il ait eu connaissance de la grande assemblée patriote qui s'est tenue à Yamachiche le 26 juillet 1837 et qui avait rassemblé des milliers de personnes ${ }^{10}$. Du moins, des proches parmi les adultes

village de Yamachiche a pu avoir très tôt des instituteurs et institutrices grâce au curé Charles Écuyer (1758-1820), ancien sulpicien, très sensible à la question de l'éducation. Voir Dionne, Antoine Gérin-Lajoie, homme de lettres, p. 41-42.

8 Casgrain, A. Gérin-Lajoie d'après ses mémoires, p. 24.

9 Albert Tessier, "Un curé missionnaire, l'abbé S.-N. Dumoulin (1793-1853)», Les Cahiers des Dix, n 16 (1951), p. 117-131. Dumoulin avait un frère député à la Chambre d'assemblée du Bas-Canada. Pierre-Benjamin Dumoulin (c17991856) a été député de Trois-Rivières de 1827 à 1832, puis de 1851 à 1854 . Il n'est pas impossible que Gérin-Lajoie l'ait connu et ait appris de lui des éléments de sa culture politique.

10 "26 juillet 1837. Assemblée de Yamachiche (Saint-Maurice)», dans Union des écrivains québécois, Assemblées publiques, résolutions et déclarations de 1837-1838, textes recueillis et présentés par Jean-Paul Bernard, Montréal, VLB éditeur, 1988, p. 156-160; Éric Bédard, Les réformistes: une génération canadienne-française au milieu du XIX siècle, Montréal, Boréal, 2009, p. 78. On évalue à 8000 personnes 
de sa famille y ont assurément assisté et ont pu en discuter devant lui, l'éveillant aux événements qui marquaient alors le pays. C'est donc dans un climat politique très lourd qu'en septembre 1837 , Antoine Gérin-Lajoie commence son cours classique en classe de syntaxe au séminaire de Nicolet.

Le directeur du Séminaire était alors Joseph-Onésime Leprohon (1789-1844), également curé de la paroisse de Nicolet, et le supérieur et procureur était l'abbé Jean Raimbault (1770-1841), un émigré de la Révolution française, qui a profondément marqué l'établissement d'un sceau de culture française d'Ancien Régime. À leur suite, le Séminaire aura un nouveau directeur, l'abbé JeanBaptiste-Antoine Ferland (1805-1865), lui-même un ancien de Nicolet et qui fut secrétaire de $\mathrm{M}^{\mathrm{gr}}$ Plessis. Il est nommé préfet des études (1841-1843), directeur (1843-1848), puis supérieur de l'établissement (1848-1850). On sait qu'il écrira, après F.-X. Garneau, un cours d'histoire du Canada ${ }^{11}$. Ferland a certainement marqué la formation intellectuelle de Gérin-Lajoie, qui deviendra son premier biographe en 1865 . Il écrira à son sujet:

Sans rompre complètement avec les traditions du passé, il sut les mettre en harmonie avec les besoins du moment; il mit en honneur les études historiques et en particulier celles du Canada. Il trouvait mille moyens d'exciter l'émulation des élèves: il en appelait à leur patriotisme, à leur honneur, cordes sensibles qui manquent rarement de vibrer dans le cœur de ces jeunes hommes chez lesquels les nobles sentiments de la nature ne sont pas encore émoussés ${ }^{12}$.

la foule qui assista à cette assemblée. Voir André Desaulniers, "Grande assemblée des patriotes à Yamachiche en 1837», Histoire d'Yamachiche, no 9 (juin 2013), [En ligne], [http://yamachiche.ca/histoire/histoire-yamachiche-juin2013.pdf] (15 janvier 2016).

${ }^{11}$ Jean-Baptiste-Antoine Ferland, Cours d'histoire du Canada: première partie: 1534-1663, Québec, Augustin Côté, 1861; seconde partie: 1663-1759, Québec, Augustin Côté, 1865.

${ }^{12}$ Antoine Gérin-Lajoie, «L'abbé J.-B.-A. Ferland», Le Foyer canadien: recueil littéraire et historique, t. III, Québec, Bureaux du Foyer canadien, 1865, p. xxx. 
Il est important de rappeler que Gérin-Lajoie est étudiant au collège lorsque les deux Canadas sont réunis et que les recommandations du rapport Durham semblent sceller le destin du Canada français. Le Ve Victis semble le sort qui attend les Canadiens ${ }^{13}$, mais dans quelques rares milieux, l'espérance n'est pas éteinte. Grâce à leur dynamisme, les leçons de l'abbé Ferland et le climat propice aux études qui régnait au séminaire de Nicolet ont pu protéger un peu Antoine Gérin-Lajoie de la morosité générale qui régnait alors dans le pays. L'abbé Ferland s'intéresse à l'éducation civique de ses élèves, et c'est grâce à lui que le jeune Gérin-Lajoie s'initie, dès ce moment-là, à l'actualité politique nationale:

Durant les récréations du soir, les élèves qui voulaient entendre parler des événements du jour se rendaient à sa chambre; et là, après leur avoir fait connaître en peu de mots les nouvelles rapportées par les derniers journaux, il prenait occasion de remonter plus haut et de rattacher à ces nouvelles les principaux événements de l'histoire moderne. C'était pour les élèves un petit cours familier d'histoire et de politique ${ }^{14}$.

Après avoir précisé, dans son éloge, que l'abbé Ferland, en commentant l'actualité, se situait bien au-dessus d'un quelconque esprit de parti, il ajoutait: «Un grand nombre d'écoliers suivaient ces leçons avec avidité et plusieurs en retirèrent des avantages incontestables ${ }^{15}$. "

Il assiste et participe aussi à l'effervescence religieuse qui accompagne la visite de $\mathrm{M}^{\mathrm{gr}}$ de Forbin-Janson au Bas-Canada. L'évêque de Nancy est reçu avec enthousiasme au séminaire de Nicolet, en février $1841^{16}$, et la retraite qu'il prêche à Trois-Rivières attire des

${ }^{13}$ Étienne Parent, rédacteur au Canadien de Québec, avait lui-même, à cette époque, désespéré de l'avenir national des Canadiens et cédé au pessimisme qui habitait les esprits entre 1839 et 1841. À Montréal, dans L'Aurore des Canadas, Joseph-Guillaume Barthe parlait des «inquiétantes destinées» qui pesaient sur l'avenir des Canadiens (L'Aurore des Canadas, 30 octobre 1841, p. 2).

${ }^{14}$ Gérin-Lajoie, «L'abbé J.-B.-A. Ferland», p. xxxi.

${ }^{15}$ Ibid.

${ }^{16}$ J.-A. Douville, Histoire du Collège-Séminaire de Nicolet 1803-1903, t. I: 18031860, Montréal, Éditions Beauchemin, 1903, p. 256. 
foules nombreuses de toute la région ${ }^{17}$. Il n'est pas interdit de croire que l'atmosphère religieuse qui marque cette époque ait pu toucher l'âme émotive de l'étudiant qu'était Antoine Gérin-Lajoie ${ }^{18}$.

Avec la sensibilité qui est la sienne, le jeune homme est attiré par la poésie. Dès l'âge de 15 ans, il compose ses premiers poèmes. Les classes de versification et des belles-lettres lui font découvrir les règles d'écriture, et il plonge dans le Cours de littérature de JeanFrançois de La Harpe (1739-1803), l'incontournable manuel de l'époque. Il consacre ses congés et ses loisirs à lire des vers. L'auteur favori de sa jeunesse est Jacques Delille (1738-1813), un poète classique de forme, mais préromantique d'esprit. En découvrant le récit de l'agonie du vieux poète aveugle, expirant dans les bras de sa compagne, Gérin-Lajoie avoue qu'il éclata en sanglots ${ }^{19}$. Il ajoute: “Je passais une grande partie de mes récréations à rêver sous les arbres ou au bord des ruisseaux. J'adorais la nature et un des plus beaux spectacles pour moi était de voir coucher le soleil ${ }^{20}$."

Pourtant, chez lui, la contemplation n'exclut pas l'action. Sa sensibilité n'est pas seulement méditative et abstraite, mais incarnée dans les destinées de ses compatriotes. En 1842, il écrit en hommage aux patriotes exilés de 1837-1838 sa fameuse complainte «Un Canadien errant", qui est publiée dans Le Charivari canadien en $1844^{21}$ et qui

${ }^{17}$ N.-E. Dionne, $M^{g r}$ de Forbin-Janson, sa vie, son auvre en Canada, Québec, Laflamme \& Proulx, 1910, p. 95-96.

18 À propos du rôle de la religion dans la sentimentalité des élèves de collèges au tournant $\mathrm{du} \mathrm{xx}^{\mathrm{e}}$ siècle, les historiennes Christine Hudon et Louise Bienvenue ont écrit: "Les élèves sont en effet mis en contact avec une religion émotive, aux inflexions doloristes, susceptible d'exacerber la sentimentalité juvénile» (Hudon et Bienvenue, Le collège classique pour garçons: études historiques sur une institution québécoise disparue, Montréal, Éditions Fides, 2014, p. 269).

${ }^{19}$ Casgrain, A. Gérin-Lajoie, p. 25-26.

20 Ibid.

${ }^{21}$ Ce journal illustré, publié de mai à octobre 1844 et imprimé à Montréal, reprochait au cabinet de Denis-Benjamin Viger sa politique de compromis avec l'administration coloniale (André Beaulieu et Jean Hamelin, La presse québécoise des origines à nos jours, t. I: 1764-1859, Sainte-Foy, Presses de l'Université Laval, 1973, p. 137). 
va connaître un immense succès ${ }^{22}$. Il a alors 18 ans, et le poème nous révèle un garçon éveillé, maîtrisant les règles de la versification et conscient des infortunes de son pays:

Si tu vois mon pays,

Mon pays malheureux,

$\mathrm{Va}$, dis à mes amis

Que je me souviens d'eux.

Il évoque aussi la nostalgie d'un temps supposé héroïque qui lui a échappé, lorsque l'exilé s’adresse à ses souvenirs : «Ô jours, si pleins d'appas/Vous êtes disparus".

Pendant l'année 1842, celle où il écrivit «Un Canadien errant", le 24 novembre, encouragé par l'abbé Ferland, il fonde, au Séminaire, une société littéraire réunissant les élèves les plus avancés du programme. Il s'agissait d'une sorte d'académie, placée sous le vocable de saint Alphonse de Ligori et fondée «sur le modèle des sociétés de discussion établies dans les grandes villes. Les membres se réunissaient une fois la semaine et passaient une heure ensemble à entendre la lecture d'un essai et à discuter une question d'histoire ou de littérature ${ }^{23}$ ". Le nombre des membres actifs de cette association était de 17 à l'origine et a, par la suite, varié de 15 à 40 membres ${ }^{24}$. C'était une sorte de laboratoire pour développer les talents oratoires et littéraires des élèves. On y appréciait les débats, et les séances étaient l'occasion d'exercices littéraires. Dans la première année d'existence de l'Académie, Antoine Gérin-Lajoie présenta une ode sur la bataille de Châteauguay, une "épître» sur l'histoire du Canada et un discours en vers sur la Résurrection du Christ ${ }^{25}$.

En 1843, avec son ami et cousin Raphaël Bellemare, comme lui étudiant, il fonde un premier journal à Nicolet, Le Moniteur. Le journal a compté 15 numéros parus entre le 15 décembre 1843 et

${ }^{22}$ Casgrain, A. Gérin-Lajoie, p. 28-29.

${ }^{23}$ Gérin-Lajoie, «L'abbé J.-B.-A. Ferland», p. xxxii.

${ }^{24}$ Douville, Histoire du Collège, p. 299.

${ }^{25}$ Ibid., p. 300. 
le 25 mai 1844. Le but de cette feuille, reproduite, semble-t-il, à la main, est «d'amuser sans être futile, d'être plus sérieux que badin, de donner le goût pour l'étude, d'inspirer l'amour du travail, d'exposer les principes de la politesse et d'encourager les entreprises utiles ${ }^{26}{ }^{\prime}$. On sent que ce garçon qui se dit lecteur contemplatif, aimant rêver sous les arbres, est aussi un hyperactif, un "pétillard», pour utiliser une de ses propres expressions, qui veut agir pour stimuler les lettres et les arts comme un engagement qui s'inscrit dans la conscience de la patrie malheureuse et menacée.

L'année suivante, il termine son cours classique et frappe un grand coup en écrivant une tragédie en vers, inspirée de l'histoire acadienne, Le jeune Latour. Il y développe l'idée de loyauté et de fidélité à la France. À certains égards, la pièce tient de la tradition classique, mais elle est déjà marquée par l'influence du courant romantique ${ }^{27}$. La pièce est montée et jouée par l'Académie du Séminaire à la fin de l'année collégiale et connaît un beau succès. Elle est publiée en septembre 1844 dans le journal montréalais de Joseph-Guillaume Barthe, L'Aurore des Canadas et, à Québec, dans Le Canadien d'Étienne Parent, ainsi que dans La Gazette de Québec. Dans le contexte social et politique de cette époque, la pièce prend un sens symbolique; elle apparaît comme une allégorie fantasmée du destin national, traçant la voie vers la réconciliation après les déchirements internes et les batailles héroïques ${ }^{28}$. Quelques années

\footnotetext{
${ }^{26}$ Beaulieu et Hamelin, La presse québécoise, p. 130; Archives du Séminaire de Québec, manuscrit 136, cité par Claude Lessard, Le Séminaire de Nicolet 18031969, Trois-Rivières, Éditions du Bien public, 1980, p. 416.

${ }^{27}$ Maurice Lemire, "Le jeune Latour", dans Maurice Lemire, Dictionnaire des cuvres littéraires du Québec, t. 1: Des origines à 1900, Montréal, Éditions Fides, 1978, p. 415-417; Micheline Cambron, "Apothéose et fin du récit romantique au Québec», dans Maurice Lemire (dir.), Le romantisme au Canada, Québec, Nuit blanche éditeur, 1993, p. 155-156, 162-163. L'auteur remercie Micheline Cambron pour ses précisions et ses conseils.

${ }^{28}$ Isabelle Décarie et Louise Frappier, "La tragédie du Jeune Latour dans Le Canadien: interférences textuelles et politiques", Études françaises, vol. 36, $\mathrm{n}^{\circ} 3$ (2000), p. $27-45$.
} 
plus tard, en 1848, elle est retenue par James Huston dans son anthologie littéraire du Canada français, Le répertoire national. La réputation littéraire du jeune auteur est dès lors reconnue.

Ceux qui ont connu Gérin-Lajoie à cette époque soulignent son talent et décrivent un jeune homme solitaire, mais aussi animé par l'enthousiasme de la jeunesse. Joseph-Guillaume Barthe, son aîné de huit ans, parle de lui en ces termes:

Il y avait alors au collège de Nicolet, parmi les rhétoriciens, un de ces êtres timides et concentrés qui semblaient avoir peur de se révéler au contact de ses condisciples et en fuyait l'occasion. Toujours replié sur lui-même, rêveur et méditatif, à l'âge où les autres sont toute pétulance et dissipation. On ne connaissait de lui que sa modestie et ses aptitudes. [...] Son caractère était mélancolique sans être sombre. [...] Deux feux sacrés couvaient dans cette âme voilée aux yeux des profanes, celui du patriotisme et de la poésie, à l'âge où d'ordinaire on ne connaît que celui de l'effervescence de la jeunesse ${ }^{29}$.

Gérin-Lajoie admettait lui-même que sa personnalité était partagée entre un côté contemplatif et un autre empressé et entreprenant: «Il y a toujours eu deux hommes en moi ", l'un plus taciturne cherche à ne pas déranger, à ne pas bousculer quoi que ce soit; l'autre est "plein d'énergie, d'enthousiasme, d'ambition, désirant les honneurs, les dangers, la gloire du monde ${ }^{30}$ ». Cette dichotomie de sa personnalité correspond bien à l'esprit du temps qui fut le sien. Comment changer le monde et s'engager dans la bataille politique quand le prix à payer risque d'être l'échec et les reculs collectifs et, sur le plan personnel, l'opprobre et l'exil? Ne vaut-il pas mieux brider les élans de sa jeunesse, accepter l'ordre des choses, travailler

${ }^{29}$ Joseph-Guillaume Barthe, Souvenirs d'un demi-siècle ou Mémoires pour servir à l'histoire contemporaine, Montréal, J. Chapleau \& fils, 1885, p. 199-201. Voir aussi le témoignage de Placide Lépine (pseudonyme de H.-R. Casgrain) dans Les Guêpes canadiennes, compilées et annotées par Aug. Laperrière, Ottawa, A. Bureau, 1881, p. 233.

${ }^{30}$ Casgrain, A. Gérin-Lajoie, p. 82, et cité dans Bédard, Les réformistes, p. 245. 
à sauver l'essentiel ou alors se donner à l'Église et à ses œuvres de miséricorde?

\section{Le goût du départ et le choc des réalités}

À la fin de son cours classique, Gérin-Lajoie a à peine 20 ans, et ses voies d'avenir sont encore bien obscures. Il est tenté par une formation en droit dans un bureau d'avocats à Montréal, mais il hésite à entrer dans cette profession encombrée, où les compromis se conjuguent avec les compromissions et où l'idéal de la justice ne s'accorde pas toujours avec les méandres du droit ${ }^{31}$. Il connaît un passage à vide d'atermoiements et de tergiversations. Il se dit «bercé d'espérances chimériques» et voudrait consacrer ses talents et ses ambitions à son pays. Il rêve de la France, où il aimerait faire une carrière dans le monde des lettres et de la politique. Il reviendrait ensuite au BasCanada, riche d'une culture et d'une expérience à mettre au service des siens en fondant à Montréal ou à Québec un journal français; il prendrait alors une part active à la politique «en ne travaillant toujours que pour l'honneur et la gloire», écrira-t-il dans ses souvenirs ${ }^{32}$.

Un ami, Guillaume Vassal, lui propose de tenter sa chance du côté des États-Unis. Il décide donc de l'accompagner avec 15 \$ en poche, espérant trouver là-bas un travail, peut-être en donnant des cours privés de français, et gagner suffisamment d'argent pour s'embarquer vers l'Europe de ses rêves. Le mythe fondateur des ÉtatsUnis l'a touché. En arrivant en terre étatsunienne, il écrit: "Nous touchions la terre classique de la liberté33!» Gérin-Lajoie quitte le Bas-Canada le 13 août 1844, mais son séjour américain est une amère déception. À bout de ressources, il doit revenir dans son

\footnotetext{
${ }^{31}$ Dans son roman Jean Rivard qu'il écrira plus tard et qui est largement inspiré de sa propre expérience, Gérin-Lajoie écrira une critique de la profession d'avocat, qu'il mettra dans la bouche du curé (Antoine Gérin-Lajoie, Jean Rivard, le défricheur, [récit de la vie réelle], suivi de Jean Rivard économiste, Montréal, Hurtubise HMH, 1976, p. 6-11).

32 Casgrain, A. Gérin-Lajoie, p. 32.

33 Ibid., p. 39.
} 
village le 30 août de la même année. En deux semaines, ses rêves de conquête se sont évanouis, et le jeune homme voit s'émousser ses nobles ambitions ${ }^{34}$.

En avril 1845, il décroche un premier emploi de réviseur au journal La Minerve, de Montréal, où Ludger Duvernay l'engage au petit salaire de $2 \$$ par semaine ${ }^{35}$. Avec ce misérable salaire, il survit plus qu'il ne vit et il est confronté aux difficultés de la vie quotidienne. Comme le Marius des Misérables, il connaît alors des jours maigres et une existence qui distille l'ambition et l'espérance. Le Bas-Canada de l'époque offre peu de promesses pour un jeune homme qui ne se destine ni à la terre ni à la vie religieuse. Pourtant, Montréal, devenue la capitale de l'Union depuis 1843, s'anime sur les plans social et culturel.

Malgré ses difficultés financières, Gérin-Lajoie multiplie les activités et s'engage pour servir son milieu. Dès 1844, il participe à la fondation de l'Institut canadien de Montréal. Il en est le premier secrétaire et est élu président en 1845-1846. Il donne à l'Institut le premier des essais publics qui y est présenté, le 16 janvier 1845: «Discours sur l'utilité des lettres». D'autres suivent: sur le commerce, en janvier 1847, un hommage posthume à Joseph-Rémi Vallières de Saint-Réal, le mois suivant et, en mai, une conférence sur les bibliothèques publiques. En même temps, il est actif au sein de la Société Saint-Jean-Baptiste de Montréal, dont il est le secrétaire en $1845^{36}$.

Il améliore son statut à La Minerve et devient chroniqueur parlementaire en 1846. Mais il délaisse un peu le journalisme pour faire ses études de droit ${ }^{37}$, malgré ses réserves initiales sur la

\footnotetext{
${ }^{34}$ Il fera en 1851 un séjour plus long aux États-Unis, à Buffalo, pour parfaire ses connaissances de l'anglais et sa compréhension de la société américaine.

${ }^{35}$ Il deviendra ensuite rédacteur, et son salaire augmentera lentement jusqu’à 5 \$ par semaine.

${ }^{36}$ Casgrain, A. Gérin-Lajoie, p. 67 ; Falardeau, "Gérin-Lajoie, Antoine».

${ }^{37}$ Il entreprend sa formation juridique, sa «cléricature» selon l'expression de l'époque, chez l'avocat Lafrenaye.
} 
profession juridique. Il est admis au Barreau en 1848, mais ne pratiquera jamais vraiment le droit. Il apprécie la doctrine, mais non la plaidoirie. Il mesure plus que jamais l'écart entre le droit et la justice et se désole de la moralité élastique de ses contemporains. En fait, sa formation juridique lui ouvrira les portes de la fonction publique, lui assurant un revenu stable et la possibilité de s'établir ${ }^{38}$.

Son idéal de carrière, il l'exprimera plus tard dans la création de son Jean Rivard, colonisateur, entrepreneur et visionnaire patriote. Si Gérin-Lajoie s'émouvait tout jeune sur les poésies de Jacques Delille, ses goûts ont évolué, et il a découvert les nouveaux modèles que sont François-René de Chateaubriand, Alphonse de Lamartine et Victor Hugo. Tous trois ont en commun d'avoir brillé sur la scène littéraire et d'avoir également mené des combats politiques. Son personnage de Jean Rivard, devenu économiste dans le second volet du roman, fera aussi une courte incursion en politique, toujours pour le service de l'intérêt supérieur de ses concitoyens.

\section{Ruptures politiques}

Gérin-Lajoie ne sent pas en lui la vocation politique, mais il ne demeurera pas entièrement en marge de l'action. Il ne sera pas député ni même candidat, mais il sera un temps militant. Dès la fin de ses études, il soutient l'action de Louis-Hippolyte LaFontaine et d'Augustin-Norbert Morin et le parti que l'on appelle bientôt les réformistes $^{39}$. En 1845, il applaudit en vers et en prose à l'amnistie des patriotes exilés et il célèbre le retour au pays du héros de sa jeunesse, Louis-Joseph Papineau. Dès janvier 1845, il écrit un poème, "Salut

${ }^{38}$ Casgrain, A. Gérin-Lajoie, p. 85; Falardeau, "Gérin-Lajoie, Antoine».

${ }^{39}$ Dès 1840, dans son Manifeste aux électeurs de Terrebonne, LaFontaine utilisait le terme «réformiste» pour désigner l'alliance électorale qu'il souhaitait avec les partisans de Robert Baldwin dans le Haut-Canada. Le terme s'est bientôt imposé pour désigner le parti qui s'est formé autour de cette alliance. Voir Yvan Lamonde et Claude Corbo (dir.), Le rouge et le bleu: une anthologie de la pensée politique au Québec de la Conquête à la Révolution tranquille, Montréal, Les Presses de l'Université de Montréal, 1999, p. 144-148. 
aux exilés", à l'occasion du retour des patriotes amnistiés ${ }^{40}$. Le texte est publié dans la Revue canadienne, et James Huston le retiendra également dans son Répertoire national. C'est une réponse à la complainte du "Canadien errant». L'œuvre s'inscrit dans une première traduction littéraire et poétique de l'histoire récente et de l'horizon politique des années $1840^{41}$. Gérin-Lajoie y appelle de ses vœux un nouveau printemps national qui serait marqué au coin de l'unité:

Retire-toi d'ici, discorde ténébreuse,

Assez longtemps ton fiel empoisonna nos jours;

Mais accours, toi, plutôt, ô paix délicieuse,

Viens unir tous les cœurs, les unir pour toujours.

Comme journaliste, il constate l'enlisement de l'Union sous l'administration du gouverneur Metcalfe et se permet une critique poétique des maigres bénéfices de l'administration de Denis-Benjamin Viger. Pour Gérin-Lajoie, le Canada vit un «hiver sombre» et de «noirs frimas [...] couvrent nos montagnes ${ }^{42}$ ». Lorsque les élections générales sont déclenchées en 1847, il pousse L.-J. Papineau à se présenter dans la circonscription de Saint-Maurice. Le 5 décembre 1847, il organise une assemblée des électeurs du comté dans son village de Yamachiche et soutient des résolutions sollicitant Papineau. Contre les prévisions des chefs du parti, Papineau accepta l'offre des citoyens et électeurs de Saint-Maurice ${ }^{43}$.

${ }^{40}$ Antoine Gérin-Lajoie, "Salut aux exilés», dans Yolande Grisé et Jeanne d'Arc Lortie (dir.), Les textes poétiques du Canada français 1606-1867, t. 4 : 1838-1849, Montréal, Éditions Fides, 1991, p. 674-677.

${ }^{41}$ Sur cette question, voir Micheline Cambron, «Du Canadien errant au Salut aux exilés: l'entrecroisement de l'histoire et de la fiction", Études françaises, vol. 27, $\mathrm{n}^{\circ} 1$ (printemps 1991), p. 75-86.

42 Gérin-Lajoie, "Salut aux exilés".

${ }^{43}$ Casgrain, A. Gérin-Lajoie, p. 79-80; sur le retour politique de Louis-Joseph Papineau, voir Jacques Monet, «La rentrée en scène de Papineau, novembre 1847mai 1848 ", dans La première révolution tranquille: le nationalisme canadienfrançais (1837-1850), Montréal, Éditions Fides, 1981, p. 349-364; LouisJoseph Papineau, "Adresse aux électeurs des comtés de Saint-Maurice et de Huntingdon, 20 décembre 1847 ", dans Cette fatale union: adresses, discours et 
Mais, dans son enthousiasme, Gérin-Lajoie a mal évalué la fracture qui se dessine rapidement entre les réformistes modérés et le groupe le plus engagé qui se solidarise avec Papineau. L'année 1848 sera celle d'un douloureux divorce politique entre les libéraux et les réformistes :

J'avais toujours admiré les talents oratoires de M. Papineau, je ressentais une espèce de vénération pour ce vieil athlète politique, et rien ne me brisa le cœur comme la nécessité de m’éloigner de lui. Mais il n'y avait pas à balancer, il fallait ou se déclarer ouvertement contre M. Papineau, ou déserter le parti de LaFontaineMorin, qui se composait de la presque totalité des Canadiens français, et sous la bannière duquel j’avais marché et combattu depuis ma sortie du collège. Dans cette alternative, je ne pouvais pas hésiter un instant, et je ne cachai pas mes sentiments ${ }^{44}$.

En effet, il brûle ses vaisseaux. Abandonnant Papineau, il rédige, en 1848 , un pamphlet contre celui qu'il saluait naguère en héros ${ }^{45}$. Il conservera un souvenir désabusé de cette fragmentation politique des partis qui, selon lui, fragilise la cause nationale. Il l'évoquera dans ses œuvres futures. Son alter ego, Jean Rivard, vivra les déceptions de la vie parlementaire et constatera que seule l'union des énergies et des opinions peut sauver le peuple et racheter la patrie. Le personnage est coincé par les tactiques de la partisanerie, laquelle rend la vie difficile à un esprit qui se veut libre, honnête et indépendant ${ }^{46}$.

manifestes 1847-1848, introduction et notes de Georges Aubin, Montréal, Lux Éditeur, 2003, p. 15-32.

${ }^{44}$ Casgrain, A. Gérin-Lajoie, p. 82-83.

${ }^{45}$ Bédard, Les réformistes, p. 244. Il s'agit vraisemblablement de la brochure "Résumé impartial de la discussion Papineau-Nelson sur les événements de Saint-Denis 1837 ", Montréal, s. é., novembre 1848, 16 p.

${ }^{46}$ Gérin-Lajoie a essentiellement développé la carrière politique de son personnage dans la première version du roman paru dans Le Foyer canadien, en 1862. Cet épisode sera retranché dans les éditions subséquentes de l'œuvre. Pour une édition qui intègre en annexe ces chapitres, on consultera l'édition publiée par Yannick Roy, A. Gérin-Lajoie, Jean Rivard, le défricheur, suivi de Jean Rivard, économiste, Montréal, Boréal compact, 2008, p. 419-[464]. 
La protection de Morin et de LaFontaine lui vaudra un poste de fonctionnaire. Le journalisme lui donnait une tribune, mais la fonction publique lui offre une stabilité financière et la chance de s'établir enfin dans la vie. Après avoir occupé un poste de copiste au ministère des Travaux publics, de "trésorier payeur", puis de traducteur, il est nommé bibliothécaire parlementaire en 1856. On a déjà considéré la bibliothèque du Parlement comme un asile pour les intellectuels de l'époque ${ }^{47}$. Casgrain écrira à ce propos: «La vie d'employé [de l'État], en lui ôtant les soucis du lendemain, devait le rendre à cette liberté d'esprit qui était pour lui la source de tant de jouissances. [...] Les loisirs qu'elle lui laissait lui permettaient de donner libre carrière à ses idées spéculatives et à s'abandonner tout entier à son amour de l'étude ${ }^{48}{ }$.

Même si ses engagements politiques lui permettent, à la fin des années 1840, d'obtenir un poste stable qui lui donne suffisamment de loisirs pour se consacrer à l'étude et à la littérature, Gérin-Lajoie reste un homme désabusé de la politique partisane. Il voit dans cette évolution une déchéance du politique. On trahit souvent l'honneur et l'intérêt supérieur de la patrie au profit des honneurs et de l'ambition personnelle. Il se désole de la corruption et des "vices», il veut faire de sa vie une sorte d'apostolat, il songe même un moment à devenir frère enseignant, puis il y renonce pour servir les siens autrement, par les lettres et l'éducation. «Il me semble voir sous un jour plus frappant, le néant des choses humaines, écrit-il, la frivolité de tout ce qui rattache les hommes à la terre, la folie de l'ambition, de la cupiditét ${ }^{49}{ }^{\prime}$.

\footnotetext{
${ }^{47}$ Alain Lacombe, Errol Bouchette: un intellectuel 1862-1912, Montréal, Éditions Fides, 1997, p. 48.

${ }^{48}$ Casgrain, A. Gérin-Lajoie, p. 85.

${ }^{49}$ Ibid., p. 97.
} 


\section{Bâtir l'idéal}

Il a toujours voulu travailler au "bonheur du peuple ${ }^{50}$ ", c'était, là aussi, le principe fondateur de l'Institut canadien: "Rendre le peuple meilleur». À la fin de la décennie, il se consacre à un travail d'éducation populaire sur le droit et sur les institutions politiques ${ }^{51}$. En décembre 1850, il écrit:

J'ai employé mes heures de loisir, depuis quelque temps, à rédiger un petit ouvrage qui contient les éléments de notre droit public et que j'ai intitulé: Catéchisme politique. Je fais imprimer cette brochure par M. Louis Perrault. J'ignore comment elle sera reçue du public. Elle ne peut froisser les sentiments de personne. Dans tous les cas, je suis sans inquiétude, ne comptant pas sur cette publication pour améliorer ma position sous le rapport pécuniaire ni sous le rapport purement social ${ }^{52}$.

Gérin-Lajoie s'adresse à la jeunesse de son temps pour lui donner un outil servant à décoder le monde dans lequel elle vit et agit:

Rendu à quinze ou vingt ans, le jeune homme lit et écoute avec une singulière avidité tout ce qui a rapport aux affaires de son pays; les assemblées publiques, la lecture des journaux intéressent sa curiosité. Mais malheureusement, l'absence de connaissances préliminaires et fondamentales rend longtemps cette curiosité presqu'infructueuse $[s i c]$, et il faut souvent l'expérience attentive de plusieurs années pour bien comprendre ce que la lecture d'un ouvrage élémentaire eût fait saisir en quelques instants. Puisse ce travail suppléer un peu à cette lacune ${ }^{53}$ !

La décennie suivante sera celle de son installation dans la vie. Il a fini de survivre à peine comme journaliste ou d'attendre des clients comme avocat. Le statut de fonctionnaire répond mieux

\footnotetext{
${ }^{50}$ Ibid., p. 86.

${ }^{51}$ Antoine Gérin-Lajoie, Catéchisme politique ou Élémens [sic] du droit public et constitutionnel du Canada, mis à la portée du peuple; avec un appendice..., Montréal, L. Perrault, 1851.

52 Casgrain, A. Gérin-Lajoie, p. 99.

${ }^{53}$ Gérin-Lajoie, Catéchisme politique, p. iii-iv.
} 
à son tempérament, et le poste de bibliothécaire parlementaire de la Législature lui convient parfaitement ${ }^{54}$. Deux ans plus tard, à 34 ans, il épouse Joséphine Parent, fille de son ami et mentor Étienne Parent. Désormais, il se rangera dans le confort d'une vie plus bourgeoise.

La décennie 1840 demeure fondamentale à ses yeux et a permis une certaine évolution de la démocratie parlementaire. À la demande de plusieurs députés, il rédige son essai d'histoire récente, Dix ans au Canada, qui ne sera publié qu'après sa mort. À propos de ce livre et de son titre, Henri-Raymond Casgrain écrivait:

Cette décade, on le sait, a fait époque dans nos annales. C'est durant cette période que s'est livrée la plus grande lutte de notre histoire moderne, la lutte pour la conquête du gouvernement responsable dont nous jouissons et dont l'établissement définitif a été le signal du progrès et du développement immenses dont nous sommes les heureux témoins ${ }^{55}$.

Pour l'auteur, ce livre, basé sur des sources publiques, était destiné à devenir un outil de référence pratique pour les «jeunes gens qui désirent prendre une part active aux affaires publiques ${ }^{56}$ ", dans le but non de servir les intérêts d'un parti, mais seulement «ceux de la justice et de la vérité ${ }^{57}$ ".

Son roman, Jean Rivard, publié en feuilleton en 1862, idéalise le citoyen qu'il aurait rêvé d'être ${ }^{58}$. Camille Roy, pour sa part, le

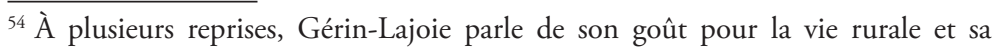
simplicité, mais les nécessités de la vie quotidienne, son besoin permanent de connaissances et de relations sociales, lui font préférer un poste comme celui de greffier, ou mieux, de bibliothécaire. Voir Casgrain, A. Gérin-Lajoie, p. 92, 102103, 114-115.

${ }^{55}$ H.-Raymond Casgrain, «Avertissement", dans Gérin-Lajoie, Dix ans au Canada, p. 7.

${ }^{56}$ Gérin-Lajoie, Dix ans au Canada, p. 10.

${ }^{57}$ Ibid., p. 9.

${ }^{58}$ Il fait naître son héros, comme lui, en 1824, dans une paroisse imaginaire, mais qu'il situe sur la rive nord du Saint-Laurent dans la vallée du lac SaintPierre. Rivard était le patronyme de sa grand-mère paternelle. Jean Rivard était 
qualifiera d'ouvrage d'économie sociale. L'œuvre d'Antoine GérinLajoie révèle l'ambition d'un homme généreux qui cherche par tous les moyens une voie de salut national, un programme énergique et optimiste malgré l'adversité. Et dans ce but, il cherche dans l'éducation et dans l'affranchissement du peuple ce qui peut préfigurer un avenir qui chante.

Il faudrait analyser cette psychologie du malheur et de l'espoir, comme celle de la «rédemption» du Canada français et les comparer aux œuvres de ceux qui, comme Gérin-Lajoie, sont nés dans les premières années 1820. Ces auteurs sont arrivés à l'âge adulte avec la lourde hypothèque de l'Union des Canadas et de la nouvelle politique coloniale de l'Angleterre, qui voyait alors le Canada français comme un obstacle au développement de son empire en Amérique du Nord. On pense à P.-J.-O. Chauveau, à Stanislas Drapeau, à Maximilien Bibaud, à Joseph-Charles Taché, à James Huston, à Thomas-Jean-Jacques Loranger, et bien d'autres. En étudiant de façon synoptique leurs trajectoires et leurs travaux, on pourrait peut-être aborder les années 1840 à la lumière de divers points de vue et voir qu'au-delà de leurs divergences idéologiques, ceux-là ont cherché une solution réaliste aux problèmes individuels et collectifs qui s’imposaient à leur génération.

Le défi était à la fois de refaire les forces d'une nation vaincue et de faire comprendre au conquérant que les conclusions de lord Durham, dans son célèbre rapport, desservaient les intérêts supérieurs de la métropole. Pour plusieurs acteurs politiques de cette période, de Louis-Hippolyte LaFontaine à Étienne-Paschal Taché, loin de nuire à l'Empire britannique en Amérique, l'existence d'un

"[...] un jeune homme sans fortune, né dans une condition modeste, qui sut s'élever par son mérite, à l'indépendance de fortune et aux premiers honneurs de son pays" (Jean Rivard, le défricheur, p. 1). Voir aussi Micheline Cambron, "Lecture et non-lecture de Jean Rivard d'Antoine Gérin-Lajoie", dans MartineEmmanuelle Lapointe et Karine Cellard (dir.), Transmission et héritages de la littérature québécoise, Montréal, Les Presses de l'Université de Montréal, 2011, p. 113-141. 
Canada français était sa meilleure garantie de se maintenir contre l'impérialisme américain. À leurs yeux, le discours national devait désormais se justifier par une acceptation de l'ordre colonial. Le loyalisme permettait d'obtenir des titres, de figurer dans les cercles du pouvoir, de réformer les institutions et, ainsi, malgré un profond désenchantement, de tracer quelques sentiers pour l'avenir. 